\title{
Normocalcemic Primary Hyperparathyroidism in Adults Without a History of Nephrolithiasis or Fractures: A Prospective Study
}

Authors

Pedro Weslley Rosário, Maria Regina Calsolari

Affiliation

Santa Casa de Belo Horizonte, Minas Gerais, Brazil

Key words

primary hyperparathyroidism, normocalcemic, secondary

hyperparathyroidism, diagnosis, prevalence

received $\quad 06.08 .2018$

accepted $\quad 11.02 .2019$

Bibliography

DOI https://doi.org/10.1055/a-0859-1020

Published online: 6.3.2019

Horm Metab Res 2019; 51: 243-247

(c) Georg Thieme Verlag KG Stuttgart · New York

ISSN 0018-5043

Correspondence

Pedro Weslley Rosário

Instituto de Ensino e Pesquisa da Santa Casa de Belo

Horizonte

Rua Domingos Vieira 590

Santa Efigênia

CEP 30150-240

Belo Horizonte

MG

Brazil

Tel.: + 55/31/32388 819, Fax: + 55/31/32388980

pedrowsrosario@gmail.com

\begin{abstract}
The prevalence and the diagnostic criterion of "normocalcemic" primary hyperparathyroidism (NPHPT) are still uncertain and there is no consensual definition. This prospective study evaluated the prevalence of NPHPT in 676 adults without a history of fractures or nephrolithiasis and who would be submitted to thyroidectomy, the impact of adopting different cut-off values for 25-hydroxyvitamin D and estimated glomerular filtration rate (eGFR), and the agreement between biochemical diagnosis and the surgical finding of altered parathyroid glands. NPHPT was diagnosed in patients with normal total and ionized calcium and elevated PTH (in 2 measurements) and without a known cause of secondary HPT, including eGFR $<40 \mathrm{ml} / \mathrm{min} /$ $1.73 \mathrm{~m}^{2}$ and 25 -hydroxyvitamin $\mathrm{D}<20 \mathrm{ng} / \mathrm{dl}$. The 4 parathyroid glands were fully explored in these patients. Forty-six patients (6.8\%) had a laboratory diagnosis of NPHPT. Altered parathyroid glands were detected in only 4 patients, corresponding to $0.6 \%$ of all patients and to $8.7 \%$ of those with a biochemical diagnosis of NPHPT. The latter was confirmed in 0/174 men, 1/252 premenopausal women, and 3/250 postmenopausal women. Among the 42 patients with elevated PTH and without altered parathyroid glands, 25 had 25-hydroxyvitamin D between 20 and $30 \mathrm{ng} / \mathrm{dl}, 7$ had eGFR between 40 and $60 \mathrm{ml} / \mathrm{min} / 1.73 \mathrm{~m}^{2}$, and 9 had both. The prevalence of NPHPT was $0.74 \%$ in this adult population without a history of nephrolithiasis or fractures. The diagnostic criterion using eGFR $>60 \mathrm{ml} / \mathrm{min} / 1.73 \mathrm{~m}^{2}$ and 25 -hydroxyvitamin D>30 ng/dl was more appropriate considering the agreement with the surgical finding of altered parathyroid glands.
\end{abstract}

\section{Introduction}

Classical and "symptomatic" primary hyperparathyroidism (PHPT) manifests with nephrolithiasis, fractures, or symptoms of hypercalcemia. Nowadays, most patients are "asymptomatic" at the time of diagnosis despite elevated serum calcium. Finally, some patients have normal calcium concentrations, which do not rule out bone or renal complications. The prevalence and even the diagnostic criterion of "normocalcemic" PHPT (NPHPT) are still uncertain and there is no consensual definition. In general, the diagnosis of NPHPT is made based on the finding of (i) normocalcemia, including ionized calcium, (ii) associated with elevated parathyroid hormone (PTH), and both should be confirmed (iii) after the exclusion of known causes of secondary HPT (SHPT) [1-5]. Surgery is not read- ily indicated in patients with NPHPT [6]. Thus, imaging methods for the detection of altered parathyroid glands initially are also not recommended $[1,3,4,7]$. The underlying parathyroid pathology (hyperplasia or adenoma) is therefore unknown at the time of diagnosis of NPHPT. Curiously, many patients first diagnosed with NPHPT do not progress to hypercalcemia or PTH elevation and spontaneous normalization of this hormone is even observed [8-10]. It is possible that some patients with a laboratory diagnosis of NPHPT actually do not have the condition and may correspond to the small percentage of normal individuals whose PTH is outside the reference range or to patients with inapparent or subclinical causes of SHPT. Finally, controversy exists regarding the exclusion of the 2 main causes of SHPT (chronic kidney disease and vitamin D insuf- 
ficiency). Some authors consider an estimated glomerular filtration rate $(e G F R)>40 \mathrm{ml} / \mathrm{min} / 1.73 \mathrm{~m}^{2}[11,12]$ for the diagnosis of NPHPT and others an $\mathrm{eGFR}>60 \mathrm{ml} / \mathrm{min} / 1.73 \mathrm{~m}^{2}[1-4,8,9,13,14]$, as well as concentrations of 25-hydroxyvitamin D $>20 \mathrm{ng} / \mathrm{dl}[4,9-11,13]$ or $>30 \mathrm{ng} / \mathrm{dl}[2,8,12,14]$.

In clinical practice, PTH is only requested in patients with altered calcemia or in the case of a strong suspicion of HPT (primary or secondary), that is, patients with osteoporosis, fractures, nephrolithiasis, or chronic kidney disease. NPHPT is therefore expected to be underdiagnosed in individuals without a known diagnosis of these conditions. The prevalence of NPHPT was investigated in some studies [8-10, 12, 14, 15]. First, many of these studies evaluated postmenopausal women $[8,12,15]$. Second, considering the diagnostic criterion above [1-5], limitations are identified in these studies: ionized calcium was not measured $[8-10,12,14]$, normocalcemia and PTH elevation were not confirmed $[9,10,12,14]$, hypercalciuria was not excluded as a cause of elevated PTH [8-10,12, 14], and the diagnosis was only considered when initial calcium $\geq 10.2 \mathrm{mg} / \mathrm{dl}$ [15]. In addition, diagnostic definition was not uniform, considering eGFR $>40 \mathrm{ml} / \mathrm{min} / 1.73 \mathrm{~m}^{2}[12]$ or $>60 \mathrm{ml} /$ $\mathrm{min} / 1.73 \mathrm{~m}^{2}[8,9,14]$ and 25-hydroxyvitamin D $>20 \mathrm{ng} / \mathrm{dl}[9,10]$ or $>30 \mathrm{ng} / \mathrm{dl}[8,12,14]$ for the exclusion of SHPT. Third, these studies did not investigate the underlying parathyroid pathology by imaging methods or surgery in most of the patients diagnosed with NPHPT [8-10, 12, 14, 15].

The objective of this prospective study was to evaluate the prevalence of NPHPT (whose definition included the measurement of ionized calcium, confirmation of normocalcemia and elevated PTH) in an adult population (including men and premenopausal women) without a history of fractures or nephrolithiasis. The study also analyzed the impact of this prevalence on the adoption of 2 different cut-offs for 25-hydroxyvitamin D and eGFR, and agreement of the laboratory diagnosis of NPHPT with the surgical finding of altered parathyroid glands.

\section{Patients and Methods}

\section{Study design}

The study was prospective. The selection criteria and study protocol were predefined, and the details are described below. The study was approved by the local Research Ethics Committee [16].

\section{Patients}

Adults (age $\geq 18$ years) with nodular thyroid disease consecutively seen by the authors between 2009 and 2014 and who would be submitted to thyroidectomy were evaluated. Patients whose thyroid nodules were detected by neck US because of a diagnosis of PHPT were not included. The following patients were also not included: patients with a history of nephrolithiasis, nephrocalcinosis and pathological fracture; andf patients with a personal or family history of multiple endocrine neoplasia or diagnosis of medullary thyroid cancer. We initially selected 676 patients [16].

The patients were submitted to the measurement of calcium (total and ionized), phosphorus, 25-hydroxyvitamin D, TSH, PTH, and urinary calcium, and creatinine. Six patients had a diagnosis of hypercalcemic PHPT and these cases have been published previ- ously [16]. None of the patients had PTH-independent hypercalcemia. The following subjects were excluded from the group of normocalcemic patients: patients using diuretics, lithium, bisphosphonates, denosuamb, recombinant PTH, and corticosteroids; patients with primary aldosteronism (investigated in the recommended situations [17]), a suspicion or known diagnosis of malabsorption syndrome; hyperphosphatemia, 25-hydroxyvitamin $\mathrm{D}<20 \mathrm{ng} / \mathrm{dl}$, eGFR $<40 \mathrm{ml} / \mathrm{min} / 1.73 \mathrm{~m}^{2}$, calcium/urinary creatine ratio $\geq 0.25$, or thyroid dysfunction. The remaining patients also underwent measurement of tissue anti-transglutaminase IgA antibodies and those with a positive result were excluded. Finally, calcium and PTH measurements were repeated in normocalcemic patients with elevated PTH and without a known cause of SHPT for confirmation of the initial result.

\section{Parathyroidectomy}

Trained surgeons performed the surgery of all patients included in the study. The surgeon was aware of the screening results and the team of surgeons was the same throughout the study. The 4 parathyroid glands were fully explored in patients with elevated PTH. The exploration was considered complete when either the 4 glands had been seen or when at least one enlarged and one normal parathyroid gland had been identified $[16,18]$. Parathyroid glands were examined based on their macroscopic appearance. We removed only the grossly abnormal parathyroid glands, that is, enlarged. Histology of the removed parathyroid glands was always obtained.

\section{Methods}

Total calcium [corrected for low albumin ( $<4 \mathrm{mg} / \mathrm{dl}$ ) using the formula: $(4$ - albumin $) \times 0.8+C a]$ (reference range: $8.4-10.4 \mathrm{mg} / \mathrm{dl})$ and urinary calcium were measured by a colorimetric method. Ionized calcium (reference range: $1.12-1.32 \mathrm{mmol} / \mathrm{l}$ ) was measured with a selective electrode and automatic correction for $\mathrm{pH}$ variation. Serum PTH was measured with a chemiluminescent assay (Immulite 2000, Diagnostic Products Corporation, Los Angeles, CA, USA), with reference values of $12-65 \mathrm{pg} / \mathrm{ml}$. Chemiluminescence assay was used to measure 25 -hydroxyvitamin D. Creatinine was measured by a kinetic colorimetric method. The eGFR was calculated using the Modification of Diet in Renal Disease Study equation. Tissue anti-transglutaminase IgA antibodies were measured by enzyme immunoassay. All measurements were obtained in the morning after fasting for approximately $10 \mathrm{~h}$, and the samples were processed and analyzed immediately after collection. Of note, there was no change in laboratory methods during the study.

\section{Results}

After the exclusion of 6 patients with hypercalcemic PHPT [16] and 176 with known causes of SHPT, 46 patients (6.8\%) had normal total and ionized calcium and elevated PTH in 2 measurements, in the absence of conditions associated with SHPT (see Methods). Hence, these patients could have a laboratory diagnosis of NPHPT.

During surgical exploration, altered parathyroid glands were only detected in 4 patients, corresponding to $0.6 \%$ of all patients and to $8.7 \%$ of those with a laboratory diagnosis of NPHPT. In these patients, the histological diagnosis was adenoma of the parathy- 
- Table 1 Sex, age, calcium, and PTH levels of the patients with NPHPT.

\begin{tabular}{|l|l|l|l|l|l|l|}
\hline Patient & Sex & Age (years) & Ca (mg/d) & Ca ionized (mmol/l) & PTH (pg/ml) & Histology (parathyroid) \\
\hline 1 & F & 44 & 10.3 & 1.3 & 76 & Adenoma (400 mg) \\
\hline 2 & F & 52 & 9.2 & 1.22 & 112 & Adenoma (850 mg) \\
\hline 3 & F & 58 & 9.5 & 1.28 & 108 & Adenoma (600 mg) \\
\hline 4 & F & 62 & 10.2 & 1.3 & 75 & Adenoma (800 mg) \\
\hline 5 & M & 50 & 9.8 & 1.28 & - \\
\hline
\end{tabular}

NPHPT: Normocalcemic primary hyperparathyroidism; Ca: Calcium; PTH: Parathyroid hormone; F: Female; M: Male.

- Table 2 Frequency of NPHPT in patients without a history of nephrolithiasis or fractures using different 25-hydroxyvitamin D and eGFR cut-offs.

\begin{tabular}{|c|c|c|}
\hline $\begin{array}{l}\text { Criterion for definition of } \\
\text { NPHPT }\end{array}$ & $\begin{array}{l}\text { Frequency of } \\
\text { laboratory } \\
\text { NPHPT }\end{array}$ & $\begin{array}{l}\text { Percentage of } \\
\text { patients with } \\
\text { laboratory NPHPT } \\
\text { and confirmed } \\
\text { parathyroid } \\
\text { pathology }\end{array}$ \\
\hline $\begin{array}{l}\text { eGFR }>40 \mathrm{ml} / \mathrm{min} / 1.73 \mathrm{~m}^{2} \text { and } \\
25 \text {-hydroxyvitamin } \mathrm{D}>20 \mathrm{ng} / \mathrm{dl}\end{array}$ & $6.8 \%(n=46)$ & $8.7 \%$ \\
\hline $\begin{array}{l}\text { eGFR }>60 \mathrm{ml} / \mathrm{min} / 1.73 \mathrm{~m}^{2} \text { and } \\
25 \text {-hydroxyvitamin } \mathrm{D}>20 \mathrm{ng} / \mathrm{dl}\end{array}$ & $4.4 \%(n=30)$ & $13.3 \%$ \\
\hline $\begin{array}{l}\text { eGFR }>40 \mathrm{ml} / \mathrm{min} / 1.73 \mathrm{~m}^{2} \text { and } \\
25 \text {-hydroxyvitamin } \mathrm{D}>30 \mathrm{ng} / \mathrm{dl}\end{array}$ & $1.8 \%(n=12)$ & $33.3 \%$ \\
\hline $\begin{array}{l}\text { eGFR }>60 \mathrm{ml} / \mathrm{min} / 1.73 \mathrm{~m}^{2} \text { and } \\
25 \text {-hydroxyvitamin } \mathrm{D}>30 \mathrm{ng} / \mathrm{dl}\end{array}$ & $0.74 \%(n=5)$ & $80 \%$ \\
\hline
\end{tabular}

NPHPT: Normocalcemic primary hyperparathyroidism; eGFR: Estimated glomerular filtration rate.

roid gland. PTH was persistently normal after surgery in these 4 patients. Sex, age, calcium and PTH levels of these patients with confirmed NPHPT are shown in $>$ Table 1. NPHPT was confirmed in $0 / 174$ men, in $1 / 252$ premenopausal women ( $0.4 \%$ ), and in $3 / 250$ postmenopausal women (1.2\%).

We evaluated 42 normocalcemic patients with elevated PTH but without altered parathyroid glands during surgery, with emphasis on eGFR and 25-hydroxyvitamin D. Estimated GFR between 40 and $60 \mathrm{ml} / \mathrm{min} / 1.73 \mathrm{~m}^{2}$ and/or 25-hydroxyvitamin D between 20 and $30 \mathrm{ng} / \mathrm{dl}$ were found in 41 patients ( 25 patients had vitamin D insufficiency, 7 had stage 3 chronic kidney disease, and 9 had both). One patient exhibited elevated PTH without a known cause of SHPT (see Methods) and without altered parathyroid glands during surgical exploration ( $\vee$ Table 1 ). This patient had a urinary calcium/ creatinine clearance ratio $>0.02$ and was submitted to $99 \mathrm{mT} \mathrm{MIBI}$ SPECT/CT and neck MRI after surgery, but both were negative. The patient currently continues normocalcemic with elevated PTH. - Table 2 shows the impact of the 25-hydroxyvitamina D and eGFR cut-offs on the prevalence of NPHPT.
- Table 3 Sex, age, calcium, and PTH levels of the patients with NPHPT (group A) and patients with elevated PTH without altered parathyroid glands and who had vitamin D insufficiency and/or stage 3 CKD (group B).

\begin{tabular}{|l|l|l|}
\hline & Group A (n=5) & Group B (n=41) \\
\hline Sex & $4 \mathrm{~F}, 1 \mathrm{M}$ & $30 \mathrm{~F}, 11 \mathrm{M}$ \\
\hline Age (years) & $44-62$ (mean: 53$)$ & $32-76$ (mean: 55$)$ \\
\hline Ca (mg/dl) & $9.2-10.3$ (mean: 9.8$)$ & $8.5-9.6$ (mean: 9.2) \\
\hline Ca ionized (mmol/l) & $1.22-1.3$ (mean: 1.28$)$ & $1.14-1.25$ (mean: 1.2) \\
\hline PTH (pg/ml) & $76-112$ (mean: 92) & $78-130$ (mean: 98) \\
\hline
\end{tabular}

NPHPT: Normocalcemic primary hyperparathyroidism; CKD: Chronic kidney disease; Ca: Calcium; PTH: Parathyroid hormone; F: Female; M: Male.

The 5 patients with NPHTP were compared with the 41 patients with elevated PTH without altered parathyroid glands during surgery and who had vitamin D insufficiency (between 20 and $30 \mathrm{ng} / \mathrm{dl}$ ) and/or stage 3 chronic kidney disease (estimated GFR between 40 and $60 \mathrm{ml} / \mathrm{min} / 1.73 \mathrm{~m}^{2}$ ). There was no difference in sex, age, and serum PTH; but calcium concentrations were higher in patients with NPHTP ( $\triangleright$ Table 3).

The 25 patients with vitamin $D$ insufficiency received supplements and were followed up every 8 weeks for dose adjustment to achieve concentrations $>30 \mathrm{ng} / \mathrm{dl}$. After 6 months, the 20 patients who achieved these concentrations in all 3 measurements had normal PTH without hypercalcemia.

\section{Discussion}

This was a prospective study that used predefined and uniformly followed selection criteria of the patients, laboratory assessment, and surgical approach [16]. In the present series, the prevalence of NPHPT was also evaluated in men and in premenopausal women, who were not included in some of the previous studies $[8,12,15]$. Regarding laboratory assessment, we emphasize that total and ionized calcium was measured and that normocalcemia and elevated PTH were confirmed for the diagnosis of NPHPT. Ionized calcium was not obtained $[8-10,12,14]$ and PTH elevation was not confirmed $[9,10,12,14]$ at diagnosis in many previous series that ana- 
lyzed the prevalence of NPHPT. In those population studies, hypercalciuria was not excluded as a cause of elevated PTH [8-10, 12, 14]. With respect to the eGFR and vitamin D cut-offs for the exclusion of SHPT, each study adopted a different criterion. In contrast, hypercalciuria and the 4 possible combinations of eGFR and vitamin $D$ concentration were evaluated in the present study. We also point out that, in contrast to the previous studies [8-10,12, 14], all participants in our series were submitted to surgical exploration of the parathyroid glands, which permitted to correlate the laboratory diagnosis of NPHPT with the confirmation of altered glands.

Using the more rigorous diagnostic criterion (eGFR $>60 \mathrm{ml}$ $\min / 1.73 \mathrm{~m}^{2}$ and 25 -hydroxivitamin D $>30 \mathrm{ng} / \mathrm{dl}$ ), we found NPHPT (confirmed during surgery) in $1.2 \%$ of 250 postmenopausal women without a history of nephrolithiasis or fractures. Also in Brazil, another study found NPHPT in $8 \%$ of 179 postmenopausal women, but all of them had been referred to a specialty center for the investigation of osteoporosis [12]. In Spain, NPHPT was diagnosed in $6 / 100$ postmenopausal women but this diagnosis was confirmed in only 4 (4\%) when reevaluated after 1 year [8]. As mentioned earlier, NPHPT might be overestimated in these series since ionized calcium was not obtained [8,12], elevated PTH was not confirmed at diagnosis [12], hypercalciuria was not excluded as a cause of this finding $[8,12]$, SHPT was only considered in the presence of eGFR $<40 \mathrm{ml} / \mathrm{min} / 1.73 \mathrm{~m}^{2}[12]$, and there was no confirmation of the underlying parathyroid pathology $[8,12]$. With opposite results, the frequency of NPHPT was only $1.5 \%$ in a large Swedish study, but only patients with calcium $\geq 10.2 \mathrm{mg} / \mathrm{dl}$ were investigated [15], and no case of NPHPT was detected among 157 postmenopausal women in an Italian series [14].

To the best of our knowledge, only 3 previous studies have evaluated the prevalence of NPHPT in men and in women before menopause $[9,10,14]$. In the first, the prevalence was only $0.4 \%$ in men $>65$ years, $0.67 \%$ in men between 18 and 65 years, and $0.35 \%$ in women between 18 and 65 years. Nevertheless, these prevalence rates might be overestimated since elevated PTH was not confirmed, hypercalciuria was not investigated for the exclusion of SHPT, and only individuals with 25 -hydroxivitamin $D<20 \mathrm{ng} / \mathrm{ml}$ were excluded [9]. Corroborating the possibility of an initial misdiagnosis of NPHPT, during long-term follow-up, the criterion of PHPT persisted in only $22 \%$ of the patients and spontaneous normalization of PTH was observed in $45 \%$ [9]. In the second study, no case was diagnosed in adult women and the prevalence was $1 \%$ in men, but again elevated PTH was not confirmed and hypercalciuria was not excluded [14]. In the present study in which elevated PTH was confirmed, hypercalciuria was excluded and 25-hydroxivitamin D> $30 \mathrm{ng} / \mathrm{ml}$ was considered for diagnosis, NPHPT was found in only $0.57 \%$ of 174 men (patient without altered parathyroid gland during surgery) and in $0.4 \%$ of 252 premenopausal women.

Another result of the present study was the implication of different eGFR and 25-hydroxyvitamin D cut-offs for the diagnosis of NPHPT. The prevalence of NPHPT was $6.8 \%$ considering eGFR > $40 \mathrm{ml} / \mathrm{min} / 1.73 \mathrm{~m}^{2}$ and 25-hydroxyvitamin D $>20 \mathrm{ng} / \mathrm{dl}$ and only $0.74 \%$ using eGFR $>60 \mathrm{ml} / \mathrm{min} / 1.73 \mathrm{~m}^{2}$ and 25 -hydroxyvitamin D> $30 \mathrm{ng} / \mathrm{dl}$. Whereas altered parathyroid glands were confirmed during surgery in only $8.7 \%$ of the patients with a diagnosis of NPHPT using the first criterion, the same was observed in $80 \%$ of patients diagnosed using the second criterion. Thus, the criterion of eGFR > $60 \mathrm{ml} / \mathrm{min} / 1.73 \mathrm{~m}^{2}$ and 25-hydroxyvitamin D > $30 \mathrm{ng} / \mathrm{dl}$ seems to be more appropriate to avoid an excessive number of false-positive results. Since patients with NPHPT can have eGFR $<60 \mathrm{ml} / \mathrm{min} / 1.73 \mathrm{~m}^{2}$ and 25 -hydroxyvitamin $\mathrm{D}<30 \mathrm{ng} / \mathrm{dl}$, to ensure that these cases will also be diagnosed, replacement therapy would be useful when vitamin D is between $20-30 \mathrm{ng} / \mathrm{dl}$, maintaining persistent levels $>30 \mathrm{ng} / \mathrm{dl}$ and observing if there is normalization of PTH (SHPT) or even the development of hypercalcemia (PHPT). In the case of eGFR $<60 \mathrm{ml} / \mathrm{min} / 1.73 \mathrm{~m}^{2}$, considering the irreversibility of this situation, follow-up verifying the evolution of calcium, PTH and correlation of eGFR with PTH may be helpful in the differential diagnosis.

Our study has limitations. Imaging methods directed at the parathyroid glands were not performed before surgery. However, these methods were found to be limited in patients with NPHPT [19], and surgical exploration by an experienced surgeon is known to be more sensitive for the identification of altered parathyroid glands. Another limitation was the lack of information about the follow-up of patients with elevated PTH and eGFR 40-60 ml/ $\min / 1.73 \mathrm{~m}^{2}$ in the absence of altered parathyroid glands during surgery. However, with this negative finding, we believe that they in fact had SHPT.

In conclusion, the prevalence of NPHPT was $0.74 \%$ in an adult population without history of nephrolithiasis or fractures. The diagnostic criterion using eGFR $>60 \mathrm{ml} / \mathrm{min} / 1.73 \mathrm{~m}^{2}$ and 25 -hydroxyvitamin D>30 ng/dl was more appropriate considering the agreement with the surgical finding of altered parathyroid glands.

\section{Funding}

This work was supported by the National Council for Scientific and Technological Development (CNPq).

\section{Conflict of Interest}

The authors declare no conflict of interest.

References

[1] Zini M, Attanasio R, Cesareo R et al. AME position statement: Primary hyperparathyroidism in clinical practice. J Endocrinol Invest 2012; 35 (7 Suppl): 2-21

[2] Eastell R, Brandi ML, Costa AG et al. Diagnosis of asymptomatic primary hyperparathyroidism: Proceedings of the Fourth International Workshop. J Clin Endocrinol Metab 2014; 99: 3570-3579

[3] Wilhelm SM, Wang TS, Ruan DT et al. The American Association of Endocrine Surgeons guidelines for definitive management of primary hyperparathyroidism. JAMA Surg 2016; 151: 959-968

[4] Khan AA, Hanley DA, Rizzoli R et al. Primary hyperparathyroidism: Review and recommendations on evaluation, diagnosis, and management. A Canadian and international consensus. Osteoporos Int 2017; 28: 1-19

[5] Babwah F, Buch HN. Normocalcaemic primary hyperparathyroidism: A pragmatic approach. J Clin Pathol 2018; 71: 291-297 
[6] Bilezikian JP, Brandi ML, Eastell R et al. Guidelines for the management of asymptomatic primary hyperparathyroidism: Summary statement from the Fourth International Workshop. J Clin Endocrinol Metab 2014; 99: 3561-3569

[7] Udelsman R, Åkerström G, Biagini C et al. The surgical management of asymptomatic primary hyperparathyroidism: Proceedings of the Fourth International Workshop. J Clin Endocrinol Metab 2014; 99: 3595-3606

[8] García-Martín A, Reyes-García R, Muñoz-Torres M. Normocalcemic primary hyperparathyroidism: One-year follow-up in one hundred postmenopausal women. Endocrine 2012; 42: 764-766

[9] Cusano NE, Maalouf NM, Wang PY et al. Normocalcemic hyperparathyroidism and hypoparathyroidism in two community-based nonreferral populations. J Clin Endocrinol Metab 2013; 98: 2734-2741

[10] Kontogeorgos G, Trimpou P, Laine CM et al. Normocalcaemic, vitamin D-sufficient hyperparathyroidism - high prevalence and low morbidity in the general population: A long-term follow-up study, the WHO MONICA project, Gothenburg, Sweden. Clin Endocrinol (Oxf) 2015; 83: $277-284$

[11] Lowe H, McMahon DJ, Rubin MR et al. Normocalcemic primary hyperparathyroidism: further characterization of a new clinical phenotype. J Clin Endocrinol Metab 2007; 92: 3001-3005

[12] Marques TF, Vasconcelos R, Diniz E et al. Normocalcemic primary hyperparathyroidism in clinical practice: An indolent condition or a silent threat? Arq Bras Endocrinol Metabol 2011; 55: 314-317
[13] Bandeira F, Griz L, Chaves N et al. Diagnosis and management of primary hyperparathyroidism - a scientific statement from the Department of Bone Metabolism, the Brazilian Society for Endocrinology and Metabolism. Arq Bras Endocrinol Metabol 2013; 57: 406-424

[14] Vignali E, Cetani F, Chiavistelli S et al. Normocalcemic primary hyperparathyroidism: A survey in a small village of Southern Italy. Endocr Connect 2015; 4: 172-178

[15] Lundgren E, Rastad J, Thrufjell E et al. Population-based screening for primary hyperparathyroidism with serum calcium and parathyroid hormone values in menopausal women. Surgery 1997; 121: 287-294

[16] Rosário PW, Mourão GF, Calsolari MR. Screening for hyperparathyroidism by measurement of calcium in patients undergoing thyroid surgery: A prospective study. Horm Metab Res 2016; 48: 673-676

[17] Funder JW, Carey RM, Mantero F et al. The management of primary aldosteronism: case detection, diagnosis, and treatment: An endocrine society clinical practice guideline. J Clin Endocrinol Metab 2016; 101: 1889-1916

[18] Denizot A, Dadoun F, Meyer-Dutour A et al. Screening for primary hyperparathyroidism before thyroid surgery: A prospective study. Surgery 2002; 131: 264-269

[19] Šiprová H, Fryšák Z, Souček M. Primary hyperparathyroidism, with a focus on management of the normocalcemic form: to treat or not to treat? Endocr Pract 2016; 22: 294-301 